\title{
General Psychiatry Restless legs syndrome following the use of ziprasidone: a case report
}

To cite: Zhu C, Bi R, Hu Y, et al. Restless legs syndrome following the use of ziprasidone: a case report. General Psychiatry 2020;33:e100112. doi:10.1136/ gpsych-2019-100112

Received 11 July 2019 Revised 07 October 2019 Accepted 28 November 2019

\section{Check for updates}

(C) Author(s) (or their employer(s)) 2020. Re-use permitted under CC BY-NC. No commercial re-use. See rights and permissions. Published by BMJ.

${ }^{1}$ Department of Sleeping Disorder, Anhui Mental Health Center, Hefei, Anhui, China ${ }^{2}$ Department of Psychiatry, Shanghai Mental Health Center, Shanghai Jiao Tong University School of Medicine, Shanghai, China

${ }^{3}$ School of Public Health, Schulich School of Medicine and Dentistry, London, Ontario, Canada

${ }^{4}$ Department of Psychiatry and Psychology, Mayo Clinic School of Graduate Medical Education, Rochester, Minnesota, USA ${ }^{5}$ Shanghai Mental Health Center, Shanghai, China

${ }^{6}$ Psychiatry Department, AtlantiCare Regional Medical Center, Atlantic City, New Jersey, USA

${ }^{7}$ Department of Psychiatry, University of Rochester Medical Center, Rochester, New York, USA

Correspondence to

Dr Yezhe Lin;

yezhe.lin@foxmail.com

\section{ABSTRACT}

Restless legs syndrome (RLS) is a common sleep-related movement disorder characterised by an uncomfortable urge to move the legs that occurs during periods of inactivity. Although there have been many case reports on antipsychotic-induced RLS, ziprasidone has never been reported as a cause of RLS. We present a case of a female patient with schizophrenia who presented with symptoms of RLS following the administration of high doses of ziprasidone added to quetiapine and valproate. The patient's symptoms of RLS occurred following the administration and titration of ziprasidone to $160 \mathrm{mg}$, and were relieved upon reducing the dose to $120 \mathrm{mg} /$ day. Other potential causative medications and differential diagnoses that could have caused similar symptoms were excluded. Clinicians should be aware of the potential for ziprasidone-induced RLS. Dopamine and serotonin interaction could be the mechanism underlying ziprasidone-induced RLS.

\section{INTRODUCTION}

Restless legs syndrome (RLS), with prevalence ranging from $2 \%$ to $15 \%$ in the general population, is a common disorder associated with many medical conditions and medications. Some second-generation antipsychotics such as risperidone, ${ }^{1}$ olanzapine ${ }^{2}$ and quetiapine ${ }^{34}$ have been reported to cause secondary RLS, whereas others have been reported to help it. ${ }^{15}$ However, no cases of RLS induced by ziprasidone were found on MEDLINE or EMBASE searches done on 25 March 2018. We report a case of RLS possibly induced by ziprasidone.

\section{CASE HISTORY}

\section{Introduction of the case}

Miss Awas a 35-year-old Chinese female patient with a history of known mild intellectual disability since childhood and an 8-month history of schizophrenia (Diagnostic and Statistical Manual of Mental Disorders, Fifth Edition). She was initially treated with risperidone titrated to $6 \mathrm{mg}$ orally per day $(3 \mathrm{mg}$ two times per day) with partial improvement but persistent active commentary auditory hallucination, disorganised behaviour and agitation. A decision was made to switch to quetiapine with a maximum dose of $800 \mathrm{mg}$ daily (400 mg orally two times per day) and $500 \mathrm{mg}$ of valproic acid daily. While taking $800 \mathrm{mg}$ of quetiapine daily, the patient still reported residual symptoms of paranoia and intermittent auditory hallucination,so we decided to put her on ziprasidone. We started with $40 \mathrm{mg}$ daily and increased to 40 mg every 3 days $(40 \mathrm{mg}-80 \mathrm{mg}-120 \mathrm{mg}-160$ $\mathrm{mg})$. She developed significant improvement of psychotic symptoms after the addition of ziprasidone. However, the night after she was titrated to $160 \mathrm{mg}$ daily $(80 \mathrm{mg}$ at lunch and $80 \mathrm{mg}$ at dinner) of ziprasidone, she developed marked, intractable discomfort in bilateral legs, which she could not describe clearly given her poor iteration. The discomfort induced an urge to move her legs, was partially relieved by walking, and worsened with resting. The symptoms were worse at night and in the afternoon. She was unable to fall asleep due to significant discomfort and kept pacing in the unit during the night. Benzodiazepine was prescribed as needed to help relieve her symptoms.

\section{Diagnosis of RLS}

A diagnosis of RLS had been made based on the diagnostic criteria from the International Restless Legs Syndrome Study Group (IRLSSG) ${ }^{6}$ Miss A's symptoms met all the criteria of this IRLSSG guideline $(5 / 5)$. As for the severity of symptoms, the patient rated her symptoms as Severe (30 points in total) using the International Restless Legs Syndrome Study Group Rating Scale (IRLS).

We also ruled out any diagnosis that could mimic symptoms of RLS, making sure that the preceding manifestations were not solely accounted for as symptoms primary to another medical or behavioural condition such as myalgia, venous stasis, leg oedema, arthritis, leg cramps, positional discomfort or habitual foot tapping.

Akathisia was ruled out on the basis of the presence of dysaesthesias in the legs rather 
than gross restlessness, and worsening symptoms at night. The neurological examination (cranial nerves, motor system, reflexes, sensation, and so on) of the patient was unremarkable, so movement disorders could be ruled out. Anaemia (complete blood count), iron deficiency (complete iron panel), diabetic neuropathy (fasting blood glucose), vitamin $B_{12}$ and folate deficiency had been ruled out. Renal function (blood urea nitrogen, creatinine), thyroid function (thyroid-stimulating hormone, FT3, FT4, T3, T4), liver function (aspartate aminotransferase, alanine aminotransferase, bilirubin, direct bilirubin, gamma-glutamyl transferase) and brain MRI were all within normal limits. Per patient and collateral, Miss A has no history of smoking, alcohol or substance use, nor history of excessive caffeine use. History or family history of similar manifestations or movement disorders was also ruled out per patient and collateral history.

\section{Management and clinical course after the onset of RLS}

As several reports have shown that RLS could be induced by quetiapine, we tapered Miss A off quetiapine and valproic acid. However, even with ziprasidone as monotreatment, her symptoms of RLS persisted.

Given the patient's amelioration of psychotic symptoms with ziprasidone, treatment with ziprasidone was maintained but with a reduced dose of $120 \mathrm{mg}$ daily ( $40 \mathrm{mg}$ three times a day during meals). The day after we reduced the dosage, the patient reported minimal discomfort in bilateral legs in the afternoon, which was relieved with massage within an hour. She reported sufficient sleep at night after tapering down the dose of ziprasidone. The patient rated her symptoms as Mild (9 points in total) at this time using the IRLS. Several days later, Miss A's psychotic symptoms were resolved, and she was thus discharged home.

\section{DISCUSSION}

The presentations of our case met the diagnostic criteria established by IRLSSG for RLS. ${ }^{6}$ We differentiated RLS from akathisia by its manifestations. However, RLS is often associated with periodic limb movement disorder (PLMD) that can only be diagnosed with polysomnography. Unfortunately, polysomnography was not able to be done due to resource limitations in the hospital,thus, the association with PLMD was unable to be ascertained. The patient developed RLS on ziprasidone dosing $160 \mathrm{mg}$ daily and symptoms were significantly relieved on reduction to $120 \mathrm{mg}$ daily (optimal effective dose), divided three times a day. Our patient developed short duration of RLS daily but was able to tolerate it.

Since ziprasidone is a minor substrate of cytochrome P450 (CYP) 3A4 and CYP1A2, and quetiapine is metabolised primarily by CYP3A4 and valproic acid by mitochondrial beta-oxidation, the drug interactions between them should be minimal.

To the best of our knowledge, considering the growing use of ziprasidone worldwide for the last two decades, no case had been reported regarding RLS and ziprasidone to date.

Dopaminergic dysfunction is considered to be a possible pathophysiological mechanism in the development of RLS, and drugs that antagonise dopamine receptors are likely to induce RLS. Antipsychotics such as ziprasidone generally have intermediate to high D2 dopamine receptor occupancy. However, within an environment of 5-hydroxytryptamine (5-HT) elevation, it was likely 5-HT1A hyperactivation or 5-HT2A antagonism, ${ }^{7}$ which potentiated the serotonin-dopamine imbalance, contributed to dopaminergic hypoactivity and RLS development in our patient. What's more, like other atypical antipsychotic agents, ziprasidone is a 5-HT2A/ dopamine D2 antagonist. However, its in vitro 5-HT2A/ D2 receptor affinity ratio is higher than that of the other first-line atypical antipsychotic agents ${ }^{8}$ (namely risperidone, olanzapine, quetiapine and aripiprazole). Moreover, ziprasidone has relatively high affinity on 5-HT1A receptor, ${ }^{9}$ acting as a partial agonist. Paroxetine worsened cases of RLS as in previous reports. It is speculated that an increased serotonergic activity leading to an indirect dopaminergic antagonism by paroxetine could be the explanation of RLS generation. ${ }^{10} 11$

It is interesting to note that as a potent dopamine blocking agent, risperidone did not induce RLS in our patient. A possible explanation could be that our patient was a fast metaboliser of risperidone, which ismetabolised by CYP2D6. Another explanation might be due to the alterations in the serotonergic neurotransmission system alone, for serotonin plays a critical role in motor function and sensory information processing. ${ }^{12}$ If that is the case, we can assume that a more potent 5-HT1A activating agents, like cariprazine, adoprazine, could be more likely to aggravate RLS symptoms than a less potent one.

In conclusion, we found a dose-related case of RLS in a patient on ziprasidone therapy. The pathophysiology remained obscure. Further studies are needed to explore the mechanism of dopamine dysfunction underlying psychotropic-induced RLS. Additionally, clinicians should be aware of the possibility that ziprasidone could develop RLS, and that the severity of RLS symptoms could be dose related. Discontinuation or tapering down the dosage may be needed to relieve the symptoms of secondary RLS.

Acknowledgements Thanks to Yanhua Zhang, Xin Cheng, Kun Cheng and Xinyi Zhang for their significant contributions towards the study.

Contributors YL was responsible for the study design and manuscript editing, literature searches and manuscript writing. BI and QL were involved in evolving the ideas and editing the manuscript. RB was involved in editing and submitting the manuscript. YH was involved in editing the manuscript. All authors have contributed to and have approved the final manuscript.

Funding This case report was funded by Psychosomatic Medicine Project of Key Developing Disciplines of Shanghai Municipal Health Commission (2019ZB0202) in China.

Competing interests None declared.

Patient consent for publication Obtained. 
Ethics approval The present study was approved by the Anhui Mental Health Center Ethics Committee, and it is conformed to the provisions of the Declaration of Helsinki.

Provenance and peer review Not commissioned; externally peer reviewed.

Open access This is an open access article distributed in accordance with the Creative Commons Attribution Non Commercial (CC BY-NC 4.0) license, which permits others to distribute, remix, adapt, build upon this work non-commercially, and license their derivative works on different terms, provided the original work is properly cited, appropriate credit is given, any changes made indicated, and the use is non-commercial. See: http://creativecommons.org/licenses/by-nc/4.0/.

\section{ORCID iD}

Ran Bi http://orcid.org/0000-0002-2917-7198

\section{REFERENCES}

1 Wetter TC, Brunner J, Bronisch T. Restless legs syndrome probably induced by risperidone treatment. Pharmacopsychiatry 2002;35:109-11.

2 Kraus T, Schuld A, Pollmächer T. Periodic leg movements in sleep and restless legs syndrome probably caused by olanzapine. J Clin Psychopharmacol 1999;19:478-9.

3 Rittmannsberger H, Werl R. Restless legs syndrome induced by quetiapine: report of seven cases and review of the literature. Int $J$ Neuropsychopharmacol 2013;16:1427-31.
4 Pinninti NR, Mago R, Townsend J, et al. Periodic restless legs syndrome associated with quetiapine use. J Clin Psychopharmacol 2005;25:617-8.

5 McLean AJ. The use of the dopamine-receptor partial agonist aripiprazole in the treatment of restless legs syndrome. Sleep 2004;27:1022.

6 Allen RP, Picchietti DL, Garcia-Borreguero D, et al. Restless legs syndrome/Willis-Ekbom disease diagnostic criteria: updated international restless legs syndrome Study Group (IRLSSG) consensus criteria - history, rationale, description, and significance. Sleep Med 2014;15:860-73.

7 Kohen I, Gordon ML, Manu P. Serotonin Syndrome in Elderly Patients Treated for Psychotic Depression with Atypical Antipsychotics and Antidepressants: Two Case Reports. CNS Spectr 2007;12:596-8.

8 Stahl SM, Shayegan DK. The psychopharmacology of ziprasidone: receptor-binding properties and real-world psychiatric practice. $J$ Clin Psychiatry 2003;64:6-12.

9 Newman-Tancredi A, Kleven MS. Comparative pharmacology of antipsychotics possessing combined dopamine D2 and serotonin 5-HT1A receptor properties. Psychopharmacology 2011;216:451-73.

10 Sanz-Fuentenebro FJ, Huidobro A, Tejadas-Rivas A. Restless legs syndrome and paroxetine. Acta Psychiatr Scand 1996;94:482-4.

11 Chou K-J, Chen P-Y, Huang M-C. Restless legs syndrome following the combined use of quetiapine and paroxetine. Prog Neuropsychopharmacol Biol Psychiatry 2010;34:1139-40.

12 Michopoulos I, Ferentinos P, Oulis P, et al. Restless legs syndrome associated with the combined use of quetiapine and venlafaxine. $J$ Clin Psychopharmacol 2014;34:159-61.

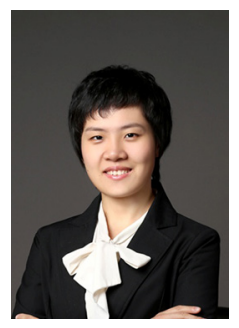

Yezhe Lin is currently a postdoctoral associate in the Department of Psychiatry at the University of Rochester Medical Center. She graduated from the Shanghai jiaotong University School of Medicine with a bachelor's degree of medicine and master's degree of psychiatry in 2014 and 2017 respectively. She also received clinical training at the Shanghai Mental Health Center from 2017 to 2018. She had acquired a bunch of research experience in metabolic side effects of antipsychotics and physical comorbidity of major psychiatric illness. Her main research interests include the pain and reward center of the brain. 